\title{
Interfacial mixing during annealing of zinc oxide nanoparticle junctions
}

\author{
Ming $\mathrm{Hu},{ }^{1, a)}$ Konstantinos P. Giapis, ${ }^{2, b)}$ and Dimos Poulikakos ${ }^{1, \mathrm{c})}$ \\ ${ }^{1}$ Department of Mechanical and Process Engineering, Laboratory of Thermodynamics in Emerging \\ Technologies, ETH Zürich, 8092 Zurich, Switzerland \\ ${ }^{2}$ Division of Chemistry and Chemical Engineering, California Institute of Technology, Pasadena, \\ California 91125, USA
}

(Received 21 February 2011; accepted 3 May 2011; published online 23 May 2011)

\begin{abstract}
The process of forming a junction between crystalline zinc oxide $(\mathrm{ZnO})$ nanoparticles during pulsed thermal annealing in liquid tetradecane is studied using molecular dynamics simulation. Pairs of equal and unequal size particles are considered with emphasis on neck growth and atom mixing. The contact area and interface width of the junction are found to increase with heat pulse power albeit at different rates. The results suggest that it is possible to increase the junction area without significant mixing of atoms across the junction interface by tailoring the heat pulse power. (C) 2011 American Institute of Physics. [doi:10.1063/1.3593487]
\end{abstract}

Nanoparticles (NPs) can serve as building blocks for generating larger structures by the so-called bottom-up approach. Joining NPs in pairs without sintering can produce anisotropic composites in terms of shape or composition. ${ }^{1}$ Oriented attachment of PbSe nanocrystals has led to junction formation with unusual optoelectronic properties. ${ }^{2}$ Exciton splitting across such junctions could create polarizable particles amenable to electrostatic manipulation and control. In the case of doped NPs, mesoscale assemblies of $p$ - $n$ nanojunctions could offer a cheaper approach to fabricating photovoltaic devices.

When solid NPs are brought together at ambient temperature, surface interactions may force them to join, creating a "neck" region. The cross-section of the junction is typically small (compared to the particle size) while minimal atomic rearrangement occurs. Selective heating of the particle pair by pulsed laser irradiation may increase atom diffusivity, enlarge the junction area, and improve connectivity. If coalescence is prevented, the anisotropic character of the composite may remain intact.

We report results from molecular dynamics (MD) simulations on the controlled annealing of zinc oxide $(\mathrm{ZnO}) \mathrm{NP}$ pairs to form nanometer-size junctions. $\mathrm{ZnO}$ was chosen because it is a wide band-gap semiconductor with potential applications in junction photovoltaics. ${ }^{3}$ However, extraneous doping is not considered in this letter to avoid the need for additional potentials. The $\mathrm{ZnO}$ NPs are thought as suspended in an inert liquid and heated by short duration heat pulses. Then, the contact area between the NPs and the interface width of the junction are calculated as a function of the heat pulse power. We seek conditions that maximize the contact area without excessive deterioration in junction abruptness though atom mixing. ${ }^{4,5}$

Our model structure consists of two spherical ZnO NPs in contact. The radius of the NPs varies from 1 to $4 \mathrm{~nm}$, corresponding to a number of $\mathrm{ZnO}$ atoms between 408 and 9456. All particles are "cut" from a perfect wurtzite $\mathrm{ZnO}$ crystal and are immersed in a liquid, ${ }^{6}$ which contains 33800

\footnotetext{
a) Author to whom correspondence should be addressed. Electronic mail: hum@ethz.ch.

b)Electronic mail: giapis@cheme.caltech.edu.

${ }^{c)}$ Electronic mail: dimos.poulikakos@ethz.ch.
}

tetradecane $\left(\mathrm{C}_{14} \mathrm{H}_{30}\right)$ molecules (473200 "united" atomsdefined as $\mathrm{CH}_{2}$ moieties-representing the full 1487200 carbon and hydrogen atoms). The entire system is contained in a cubic simulation box with size $24.28^{3} \mathrm{~nm}^{3}$. Periodic boundary conditions were used in all directions.

All MD simulations were performed with the LAMMPS package. ${ }^{7}$ A time step of 1 fs was used throughout the simulation. The van der Waals forces were truncated at $1 \mathrm{~nm}$. Long-range Coulombic interactions were computed using the particle-particle, particle-mesh algorithm. ${ }^{8}$ For the $\mathrm{ZnO}$ interatomic potential, we opted for the core-shell model, ${ }^{9}$ which has been widely used to predict the mechanical and thermal properties of $\mathrm{ZnO}$ in different phases. ${ }^{10-12}$ The Buckingham potential were truncated at $1.5 \mathrm{~nm}$. Interactions between united atoms forming the tetradecane molecules were described by the Amber force field ${ }^{13}$ with all nonbonded interaction energies calculated from the Lennard-Jones potential, $e(r)=4 \varepsilon\left[(\sigma / r)^{12}-(\sigma / r)^{6}\right]$. As usual, the energy and distance parameters $\varepsilon_{i j}$ and $\sigma_{i j}$ between the $\mathrm{ZnO}$ and the tetradecane united atoms were obtained from the geometric and arithmetic mean, respectively, of the corresponding values for intermolecular interactions, listed in Ref. 6.

To relax the NP, we ran the simulation with free boundary conditions in all directions, corresponding to zero pressure and constant temperature of $T=300 \mathrm{~K}$ for $2 \mathrm{~ns}$ using a Nosé-Hoover temperature thermostat. ${ }^{14}$ After the constant temperature relaxation, we continued to relax the system with NVE (constant volume and no thermostat) ensemble for $1 \mathrm{~ns}$. Translational and rotational movement of the particles was prevented by removing the corresponding momenta.

We first computed the melting temperature of NPs with sizes ranging from 1 to $4 \mathrm{~nm}$. The temperature was first raised to $2000 \mathrm{~K}$ in $2 \mathrm{~ns}$, followed by steps of $25 \mathrm{~K}$, each of $1 \mathrm{~ns}$ duration, to avoid particle superheating. The melting temperature was determined by a jump in the total energy, combined with evidence of structural changes. The NPs melt when heated between 2200 and $2600 \mathrm{~K}$, depending on particle size, as quantified in Fig. 1. The melting temperature exhibits a strong size-dependence for particle radii between 1 and $3 \mathrm{~nm}$, beyond which it approaches gradually the bulk value. Note that the computed melting point of bulk wurtzite $\mathrm{ZnO}$ is $\sim 18 \%$ higher than the experimental value of 2250 $\mathrm{K} .{ }^{15}$ This discrepancy originates in the core-shell potential, 


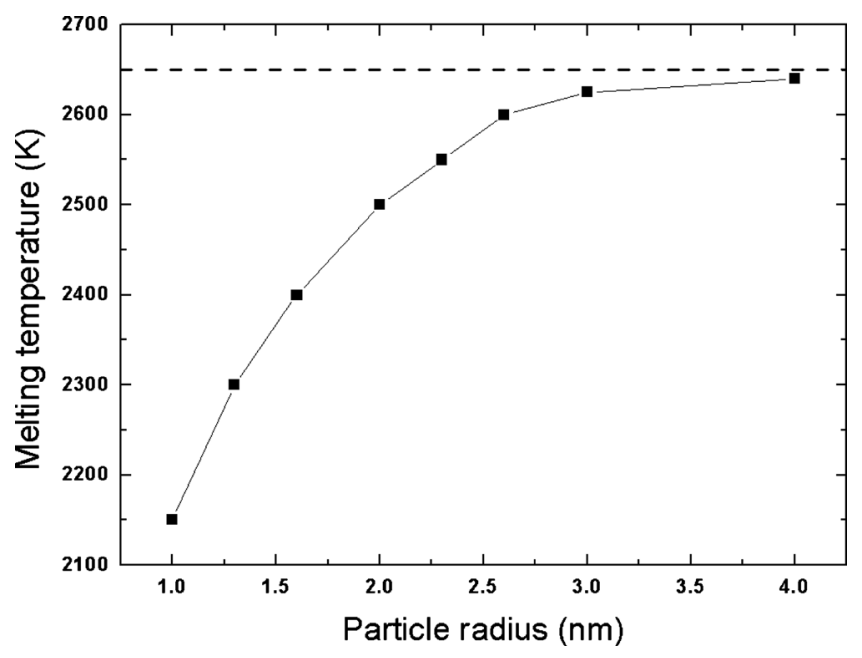

FIG. 1. Calculated melting temperature of $\mathrm{ZnO}$ NPs as a function of particle size. The dashed line is the computed bulk melting point.

which predicts a very high cohesive energy of $\sim 39 \mathrm{eV},{ }^{11}$ compared to a measured $7.52 \mathrm{eV}$ for the bulk, ${ }^{16}$ leading to a very stable wurtzite structure. No experimental values for the melting point of $\mathrm{ZnO}$ NPs could be found.

The main hypothesis in this letter is that nanojunctions between particles can be improved by taking advantage of the size-dependent melting behavior. Controlled heating (e.g., by laser pulses) of proximal NPs of different size may induce melting of only the smaller particle. Wetting of the solid particle and increased atom mobility at the interface should then enlarge the contact area. If mixing of the atoms across the interface can be minimized, an abrupt and, thus, high-quality junction may be possible.

Two sets of NP pairs were subjected to the heating/ cooling cycle; (1) particles with radii of 1.6 and $3 \mathrm{~nm}$ and (2) particles of equal radius of $3 \mathrm{~nm}$. Both sets were heated in liquid tetradecane. To emulate a heat pulse, a specific amount of nontranslational kinetic energy was added to the $\mathrm{ZnO}$ atoms such that their aggregate momentum was conserved. During the entire heating/cooling cycle, the pressure and temperature in the outermost region of the simulation domain - at a radial distance greater than $11 \mathrm{~nm}$ from the NP center-was maintained at 1 atm and $300 \mathrm{~K}$, respectively.

The contact area and interface width at the particle junction after annealing were calculated by first establishing the $\mathrm{Zn}$ and $\mathrm{O}$ "atoms-in-contact," defined on the basis of the distance between them being less than or equal to the nearest neighbor distance between $\mathrm{Zn}$ and $\mathrm{O}$ atoms in the bulk $(0.2025 \mathrm{~nm})$. Then, an interface plane was determined by minimizing the distance to all the atoms-in-contact by leastsquares fitting. After projecting the atoms onto the interface plane (Fig. 2, middle inset), the contact radius was calculated from the in-plane gyration radius defined as $R_{g}^{2}=\sum_{i} m_{i}\left(\boldsymbol{r}_{i}\right.$ $\left.-\boldsymbol{r}_{0}\right)^{2} / \sum_{i} m_{i}$, where $m_{i}$ is the atomic mass, $\boldsymbol{r}_{i}$ and $\boldsymbol{r}_{0}$ the atomic coordinates, and the mass center of the projected atoms, respectively. The interface width is equivalent to the out-ofplane gyration radius, defined as $d_{g}=d_{L}+d_{R}$. Here $d_{L}$ and $d_{R}$ are the gyration distance of atoms-in-contact on the left and right side of the interface plane (Fig. 2, right inset), respectively, which is defined as, $\left[\Sigma_{d_{L, R}=} m_{i} d_{i}^{2} / \Sigma_{i} m_{i}\right]^{1 / 2}$, where $d_{i}$ is the distance of atom $i$ from the interface plane. Note that we distinguish between left and right $\mathrm{ZnO}$ particles only when determining the atoms-in-contact.

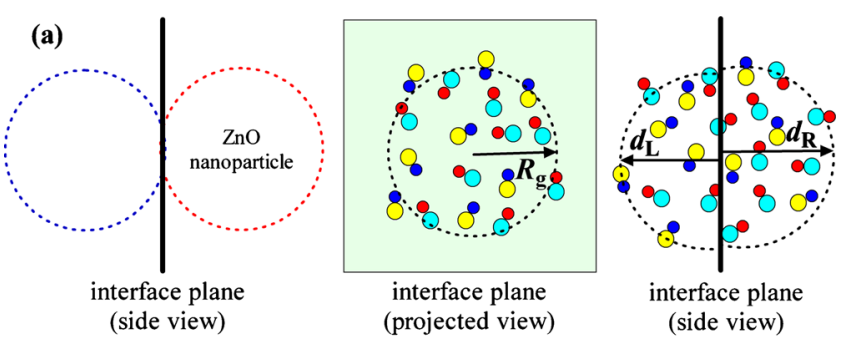

(b)

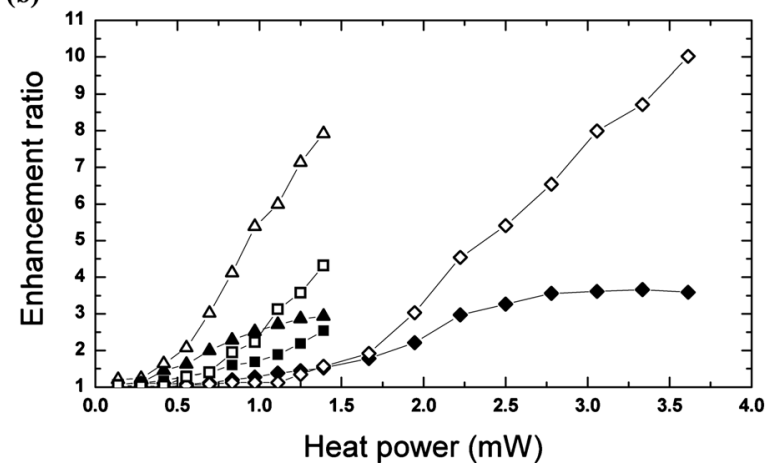

FIG. 2. (Color online) (a) Left: schematic of two NPs in contact; middle: contact radius determination based on the projection of atoms-in-contact on the interfacial plane; right: interface width determination from the left and right gyration distance. Color code: yellow and cyan: $\mathrm{Zn}$, blue and red: O. For black and white print, large light circles: Zn, small dark: O. (b) Enhancement ratio of the contact radius and interface width for different sets of $\mathrm{ZnO}$ NPs annealed in liquid tetradecane. Filled symbols refer to the contact radius; open symbols indicate the interface width. $\boldsymbol{\Delta}, \triangle 1.6 / 3.0 \mathrm{~nm}$ particle pair with same orientation; $\square, \square 1.6 / 3.0 \mathrm{~nm}$ particle pair with the small particle rotated $90^{\circ}$ along the y-direction; $\diamond, \diamond$ apply to the $3.0 / 3.0 \mathrm{~nm}$ pair.

Figure 2(b) describes how the junction area and interface width of the $\mathrm{ZnO}$ NP pairs vary as a function of heat pulse power. The results are presented in terms of the ratio of the contact area radius and interface width after the heating/ cooling cycle over their respective values before annealingtermed "enhancement ratios." The contact radius and interface width for $1.6 / 3.0 \mathrm{~nm}$ pair before heating is $6.28 \AA$ and $1.84 \AA$, respectively; these values are $7.36 \AA$ and $2.34 \AA$ for 3.0/3.0 nm pair. Results for two different crystal orientations are shown; (1) the two particles are attached with the same [0001] orientation and (2) the small particle is rotated by $90^{\circ}$ along the y-direction. The general trends are similar for both sets of NPs, regardless of orientation; the contact area and interface width both increase with increasing power. However, the interface width, exhibits greater variation for some initial orientations. Other initial orientations have been tried, e.g., rotating the small particle by $45^{\circ}$ and $90^{\circ}$ along the z-direction with no significant change in the enhancement ratio curves.

The movement of atoms at the interface can be classified into three distinct regimes. For low heat pulse power up to a threshold value, the enhancement seen for the contact radius is virtually identical to that for the interface width. This regime corresponds to the uniform expansion of the necking region between the particles, driven by large atomic potential gradients. ${ }^{17,18}$ The driving mechanism in this regime is surface diffusion, leading to atom accumulation at the sharp cusp; the more energy the atoms absorb, the larger the adjoining area grows. ${ }^{18}$ No dislocation or slip was found in this regime. Selected cases were recalculated with different initial velocities to assess the sensitivity of the results on initial 

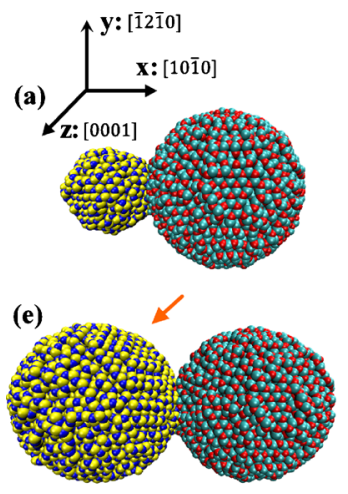

(b)

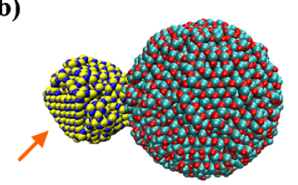

(f)

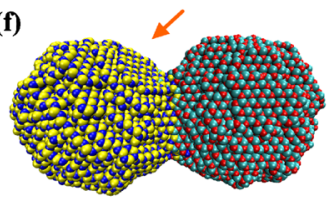

(c)

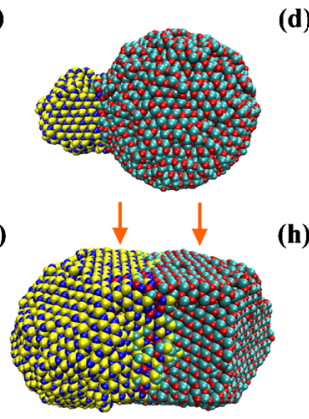

(d)

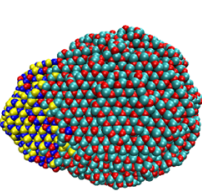

(h)

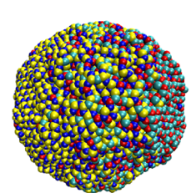

FIG. 3. (Color online) Crystallized configurations of joined $\mathrm{ZnO}$ NPs heated in liquid tetradecane at the indicated heat pulse powers. Each structure is shown in the [0001] projection. The arrows indicate the close-packed plane of the HCP structure. [(a)-(d)]: $R=1.6 / 3 \mathrm{~nm}$ pair; $\quad[(\mathrm{e})-(\mathrm{h})]: \quad R$ $=3 / 3 \mathrm{~nm}$ pair. Pulse power (in $\mathrm{mW}$ ): (a) 0, (b) 0.28 , (c) 0.56, (d) 0.97, (e) 0, (f) 1.67, (g) 2.22, (h) 3.61. Same color code as Fig. 2. conditions. In all cases tried, the results were within $7 \%$ of those reported in Fig. 2(b).

As the heat pulse power increases, the enhancement ratios diverge; the junction interface width increases much more than the contact area radius. It must be noted that the point of divergence does not always correspond to NP melting. For the $1.6 / 3.0 \mathrm{~nm}$ pair, the smaller particle melts but the larger particle is still solid at the divergence point of $0.42 \mathrm{~mW}$. For the $3.0 / 3.0 \mathrm{~nm}$ pair, however, both NPs are solid at the corresponding divergence point of $1.67 \mathrm{~mW}$ (in this case melting occurs at $3.61 \mathrm{~mW}$ ). The contact area radius continues to increase slowly for larger pulse power until it reaches a plateau. In contrast, the interface width increases steeply with increasing pulse power, consistent with increased atom diffusivity at higher temperatures. In this regime, grain-boundary diffusion is believed to dominate the movement. As pointed out in Ref. 17, grain-boundary and surface diffusion are the major mechanisms for neck growth before melting for particles less than $10 \mathrm{~nm}$. The contact radius and interface width-dependence on heat pulse power suggests that, in order to make high quality $p-n$ junctions, the NPs should be heated in the linear regime. Beyond that, significant mixing at the interface will degrade the abruptness of the junction.

As the heat pulse power is increased further, both NPs melt. Here we focus our analysis on the 3.0/3.0 nm pair. We cannot achieve melting of both particles in the $1.6 / 3.0 \mathrm{~nm}$ pair because the small particle is decomposed before the larger NP melts. When both NPs melt, sintering and subsequent coalescence occurs. ${ }^{6,19}$ This process is finished in the first 50-100 ps, producing a single mixed particle with a very wide interfacial region due to the much larger atom diffusivity in the melt.

Selected final configurations of the joined NPs after annealing at typical heat pulse powers are shown in Fig. 3. In both cases, a neck forms between the two particles at low power. When heated to an elevated temperature, the hexagonal closed packed (HCP) surface of a perfect wurtzite becomes easily discerned [Fig. 3(b) and 3(f)], as expected because it has the lowest potential energy among all phases of $\mathrm{ZnO}$. As pulse power is raised, the neck grows [see Fig. 3(c) and $3(\mathrm{~g})]$ but at the same time interfacial mixing increases as well. The final configuration of the $3.0 / 3.0 \mathrm{~nm}$ pair shows a markedly different surface morphology. The composite loses its dual-particle character while its surface becomes terminated by large close-packed planes [Fig. 3(g)]. Unlike liquids, it is possible to have a nonspherical final shape in solids because the driving force (surface energy gradient) toward a spherical shape (minimum surface energy) may not be large enough to overcome the resistance posed by the crystalline structure. In that case, a rearrangement of the lattice structure occurs. At very high pulse power, the smaller particle of the $1.6 / 3.0 \mathrm{~nm}$ pair becomes incorporated into the larger one [Fig. 3(d)] while both NPs of the 3.0/3.0 nm pair melt and coalesce into a single particle [Fig. 3(h)].

In summary, junction formation between $\mathrm{ZnO}$ NP pairs suspended in a liquid was simulated, following rapid annealing. Detailed neck growth mechanisms were identified and analyzed for two different sets of particle sizes. Increase in the junction area was possible upon controlled heating without significant mixing of atoms at the junction interface.

K.P.G. is grateful to ETH Zurich for a visiting professorship grant. We acknowledge computational support from the Swiss National Supercomputing Centre-CSCS (Project No. s243) and the Brutus Cluster at ETH Zurich.

${ }^{1}$ D. V. Talapin, C. T. Black, C. R. Kagan, E. V. Shevchenko, A. Afzali, and C. B. Murray, J. Phys. Chem. C 111, 13244 (2007).

${ }^{2}$ P. Schapotschnikow, M. A. van Huis, H. W. Zandbergen, D. Vanmaekelbergh, and T. J. H. Vlugt, Nano Lett. 10, 3966 (2010).

${ }^{3}$ L. Zhuang and K. H. Wong, Appl. Phys. A: Mater. Sci. Process. 87, 787 (2007).

${ }^{4}$ N. H. Nguyen, R. Henning, and J. Z. Wen, J. Nanopart. Res. 13, 803 (2011).

${ }^{5}$ V. N. Koparde and P. T. Cummings, ACS Nano 2, 1620 (2008).

${ }^{6} \mathrm{M}$. Hu, D. Poulikakos, C. P. Grigoropoulos, and H. Pan, J. Chem. Phys. 132, 164504 (2010).

${ }^{7}$ S. Plimpton, J. Comput. Phys. 117, 1 (1995).

${ }^{8}$ P. S. Crozier, R. L. Rowley, and D. Henderson, J. Chem. Phys. 114, 7513 (2001).

${ }^{9}$ F. Oba, I. Tanaka, S. R. Nishitani, H. Adachi, B. Slater, and D. H. Gay, Philos. Mag. A 80, 1567 (2000).

${ }^{10}$ Y. Sato, T. Mizoguchi, F. Oba, Y. Ikuhara, and T. Yamamoto, Phys. Rev. B 72, 064109 (2005).

${ }^{11}$ X. W. Sun, Z. J. Liu, Q. F. Chen, H. W. Lu, T. Song, and C. W. Wang, Solid State Commun. 140, 219 (2006).

${ }^{12}$ X. W. Sun, Y. D. Chu, T. Song, Z. J. Liu, L. Zhang, X. G. Wang, Y. X. Liu, and Q. F. Chen, Solid State Commun. 142, 15 (2007).

${ }^{13}$ W. D. Cornell, P. Cieplak, C. I. Bayly, I. R. Gould, K. M. Merz, D. M. Ferguson, D. C. Spellmeyer, T. Fox, J. W. Caldwell, and P. A. Kollman, J. Am. Chem. Soc. 117, 5179 (1995).

${ }^{14}$ S. Nosé, J. Chem. Phys. 81, 511 (1984); W. G. Hoover, Phys. Rev. A 31, 1695 (1985)

${ }^{15}$ G. Guisbiers and S. Pereira, Nanotechnology 18, 435710 (2007).

${ }^{16} \mathrm{P}$. Erhart, N. Juslin, O. Goy, K. Nordlund, R. Müller, and K. Albe, J. Phys.: Condens. Matter 18, 6585 (2006).

${ }^{17}$ H. Pan, S. H. Ko, and C. P. Grigoropoulos, J. Heat Transfer 130, 092404 (2008).

${ }^{18}$ P. Zeng, S. Zajac, P. C. Clapp, and J. A. Rifkin, Mater. Sci. Eng., A 252, 301 (1998).

${ }^{19}$ S. Arcidiacono, N. R. Bieri, D. Poulikakos, and C. P. Grigoropoulos, Int. J. Multiphase Flow 30, 979 (2004). 